\title{
Nomogram Predicting Overall Survival of Resected Locally Advanced Rectal Cancer Patients with Neoadjuvant Chemoradiotherapy
}

This article was published in the following Dove Press journal: Cancer Management and Research

\author{
Jianyuan Song $\mathbb{B D}^{1-3}$ \\ Zhuhong Chen' \\ Daxin Huang' \\ Yimin $\mathrm{Wu}^{\prime}$ \\ Zhuangbin Lin ${ }^{1,2}$ \\ Pan $\mathrm{Chi}^{4}$ \\ Benhua $X u^{1-3}$
}

'Department of Radiation Oncology, Fujian Medical University Union Hospital, Fuzhou, Fujian Province, People's Republic of China; ${ }^{2}$ Department of Oncology, Fujian Medical University Union Clinical Medicine College, Fuzhou, Fujian Province, People's Republic of China; ${ }^{3}$ Department of Medical Imaging Technology, College of Medical Technology and Engineering, Fujian Medical University, Fuzhou, Fujian Province, People's Republic of China; ${ }^{4}$ Department of Colorectal Surgery, Fujian Medical University Union Hospital, Fuzhou, Fujian Province, People's Republic of China
Correspondence: Pan Chi

Department of Colorectal Surgery, Fujian Medical University Union Hospital, Fuzhou 35000I, People's Republic of China

Email chipan163163@163.com

Benhua Xu

Department of Radiation Oncology, Fujian Medical University Union Hospital, Fuzhou 35000I, People's Republic of China

Email benhuaxu@I63.com
Purpose: The overall survival (OS) of resected locally advanced rectal cancer patients who underwent neoadjuvant chemoradiotherapy (nCRT) was significantly different, even among patients with the same tumor stage. The nomogram was designed to predict OS of rectal cancer with nCRT and divide the patients into different risk groups.

Materials and Methods: Based on materials from 911 rectal cancer patients with nCRT, the multivariable Cox regression model was carried out to select the significant prognostic factors for overall survival. And then, the nomogram was formulated using these independent prognostic factors. The discrimination of the nomogram was assessed by concordance index (C-index), calibration curves and time-dependent area under curve (AUC). The patients respective risk scores were calculated through the nomogram. The best cut-off risk score was calculated to stratify the patients. The survival curves of the two different risk cohorts were performed, which assessed the predictive ability of the nomogram.

Results: Age, cT stage, pretreatment CEA, pretreatment CA19-9, surgery, posttreatment CEA, posttreatment CA19-9, pT stage, $\mathrm{pN}$ stage and adjuvant chemotherapy were selected for the construction of the nomogram. And then the nomogram was constructed with independent prognostic factors. The C-index of the nomogram was 0.724 , which showed the nomogram provided good discernment. The acceptable agreement between the predictions of nomogram and actual observations was illustrated by calibration plots for 3-, 5- and 10-year OS in the cohort. Time-dependent AUC with 6-fold cross-validation also showed consistent results of the nomogram. Risk group stratification confirmed that the nomogram had great capacity for distinguishing the prognosis.

Conclusion: The nomogram was developed and validated to predict overall survival of resected locally advanced rectal cancer patients with nCRT. The proposed nomogram might help clinicians to develop individualized treatment strategies. However, further studies are warranted to optimize the nomogram by finding out other unknown prognostic factors, and more external validation is still required.

Keywords: rectal cancer, nomogram, overall survival, neoadjuvant chemoradiotherapy

\section{Introduction}

Colorectal cancer is one of the most prevalent malignancies through the world. ${ }^{1}$ Among the locally advanced rectal cancer, neoadjuvant chemoradiotherapy (nCRT) followed by radical surgery has become the standard treatment. ${ }^{2-5}$ Various prognostic factors affect the survival outcomes of rectal cancer patients. The American Joint Committee (AJCC) on Cancer TNM staging system is most commonly used in clinical practice. However, survival heterogeneity is frequently present among 
patients with the same AJCC stage, ${ }^{6}$ which indicates that TNM staging system is not that perfect for predicting prognosis and clinical management of rectal cancer. ${ }^{6}$ A nomogram with a variety of factors is a graphic calculating scale designed to assist in diagnosis or predict the incidence and progression of the disease. A number of nomograms concerning colorectal cancer have been reported, due to the friendly user interface and nice statistical capabilities in predicting individualized outcome. ${ }^{2,7}$ Among the nomograms related to rectal cancer with nCRT, few are related to prognosis. In the present study, the nomogram was formulated to provide more individualized prognosis of locally advanced rectal cancer (LARC) with nCRT, which might contribute to process informed management and intelligent decisions for clinicians and patients.

\section{Materials and Methods}

\section{Ethics Statement}

In this study, all information was retrospectively extracted in the context of compliance with the ethical standards of the institutional and/or national research committees. Patients' medical records were analyzed retrospectively. No individual identifiable information was used. Thus, the Fujian Medical University Union Hospital Ethic Review Board approved this study.

\section{Patient Selection}

In this study, all information was obtained from the database of our institution between September 2006 and October 2017. The inclusion criteria were as follows: (a) clinical stage II or $ш$; (b) age at diagnosis $\geq 18$ years; (c) histologically proven rectal adenocarcinoma; (d) patients who underwent nCRT and surgical resection. The exclusion criteria were as follows: (a) histologically proven signet-ring cell carcinoma or other carcinoma; (b) age at diagnosis < 18 years; (c) previous or concurrent other malignancies; (d) patients with inadequate clinicopathological information. The clinicopathological characteristics include age, gender, levels of carcinoembryonic antigen (CEA), levels of carbohydrate antigen 19-9 (CA19-9), therapeutic measure, pathological tumor-nodemetastasis staging (pTNM), clinical tumor-nodemetastasis staging (cTNM), the total number of examined lymph node (TLN) and tumour location. The pretreatment parameters were defined as the levels one week before the nCRT, and the posttreatment parameters were deemed as the levels one week before surgery. Patients were staged on the basis of the AJCC staging eighth edition. Clinical $\mathrm{T}$ and $\mathrm{N}$ stages were classified on the basis of endoscopic ultrasonography and magnetic resonance imaging. Cancer location was categorized as low ( $<5 \mathrm{~cm}$ from the anorectal verge), middle $(5-10 \mathrm{~cm}$ from the anorectal verge) or high $(10-15 \mathrm{~cm}$ from the anorectal verge). ${ }^{8}$ The CEA level was categorized as normal $(<5.0 \mathrm{ng} / \mathrm{mL})$ or elevated $(\geq 5.0 \mathrm{ng} / \mathrm{mL})$. The CA19-9 level was categorized as normal $(<37 \mathrm{U} / \mathrm{mL})$ or elevated $(\geq 37 \mathrm{U} / \mathrm{mL})$.

\section{Neoadjuvant Chemoradiotherapy}

All patients received nCRT, and the specific protocol of radiotherapy has been published previously. ${ }^{9}$ The neoadjuvant radiotherapy regimen consists of 3-dimensional conformal radiotherapy (3D-CRT) and intensity-modulated radiation therapy (IMRT). The radiation dose of PTV of CTV was 45 Gy with 25 fractions. The radiation dose of PTV of GTV was $50.4 \mathrm{~Gy}$ in 28 fractions with 3DCRT or 50 Gy in 25 fractions with IMRT. The chemotherapeutic regimens included Capecitabine, CapeOX, FOLFOX4 and mFOLFOX6. Capecitabine was taken with the dosage of $825 \mathrm{mg} / \mathrm{m}^{2}$ twice daily. And the other specific protocol of chemotherapy has also been reported previously ${ }^{10}$ The capecitabine or 5-FU based neoadjuvant chemotherapy was initiated on the first day of radiotherapy.

\section{Surgery}

Surgery was carried out by experienced experts from our centre 6-12 weeks after nCRT. The operation was performed followed the tumor-specific mesorectal excision or total mesorectal excision principle, including abdominoperineal resection (APR) and other resection.

\section{Follow-Up}

The frequency of regular follow-up was once every three months within the first two years, once every six months in the next three years, and annually thereafter. Follow-ups were either by inpatient department or outpatient department review or by telephone or mail or letter. The last follow-up was completed in December 2019. Overall survival (OS) was defined as the period from the date of diagnosis to death from any cause or the last follow-up. When patients were alive or died without evidence of rectal cancer at the last follow-up, the patients were censored. 


\section{Nomogram and Data Analysis}

The least absolute shrinkage and selection operator (LASSO) regression algorithm was applied to identify the OS-related characteristics. The 10 folds LASSO regression analysis was performed with the "cv.glmnet" function of $\mathrm{R}$ package "glmnet". Where features are selected, the value $\log (\lambda)$ was set using the minimum criteria. These features were selected for the construction of the nomogram. The discrimination of the nomogram was evaluated by concordance index (C-index) and calibration curves. The $\mathrm{C}$-index was calculated. The calibration curves were constructed with a 10 -fold crossvalidation procedure by using the "calibrate" function of R package "hdnom". The validation of the nomogram was assessed by 6-fold cross-validation with timedependent area under curve (AUC) with the "validate" function of R package "hdnom". Each characteristic variable was given a point from points scale. Then, the total score was obtained by adding up each variable point. The probability of 3-, 5- and 10-year OS was obtained by locating the total score onto the total points scale. The total score of each patient was obtained by using the "nomogramEx" function of R package "nomogramEx". The best cut-off risk score was calculated by using the "surv_cutpoint" function of $\mathrm{R}$ package "survminer". Patients were assigned to the low-risk group and the high-risk group on the basis of the cut-off score. The Kaplan-Meier method was carried out to produce the survival curves of the two different risk groups. Statistical analysis were performed with $\mathrm{R}$ software 4.0.1 and the SPSS software 25.0 (SPSS Inc, Chicago, Illinois, USA).

\section{Results}

\section{Clinical Characteristics}

In total, 911 LARC patients with nCRT were enrolled. Among these patients, 595 (65.3\%) were male, and 316 $(34.7 \%)$ were female. The mean age was 55.3 years, 340 (37.3\%) patients were 60 years of age or older. The percentage of clinical positive lymph node was $90.1 \%$. The percentage of pretreatment normal CEA and pretreatment CA199 was $56.3 \%$ and $86.3 \%$, respectively. One hundred and seventy-eight (19.5\%) patients got pCR. Four hundred and twenty (46.1\%) patients did not achieve the examination of at least 12 lymph nodes. Median follow-up time was 51.4 months. The more detailed characteristics are shown in Table 1.

\section{Feature Selection and Nomogram Construction for OS}

The clinicopathological characteristics include gender, age, cT stage, cN stage, pretreatment CEA, pretreatment CA19-9, cancer location, chemotherapy classification, radiotherapy classification, surgery classification, posttreatment CEA, posttreatment CA19-9, pT stage, pN stage, TLN, adjuvant chemotherapy. These 16 features were considered as potential predictors. LASSO regression algorithm was applied for feature selection. The most appropriate tuning parameter $\lambda$ for LASSO regression was 0.010 , when the partial likelihood binomial deviance reached its minimum value (Figure 1A), 10 variables with nonzero coefficients were retained in the LASSO analysis (Figure 1B). As shown in Figure 2, all selected variables were combined to formulate a nomogram which predicts the 3-, 5- and 10-year OS. The nomogram illustrated that $\mathrm{pN}$ classification, $\mathrm{pT}$ classification and posttreatment CA199 contributed the most to prognosis. Each subtype of these variables was assigned a score on the point scale. The survival probability of the individual patients was predicted though the score addition of all the selected variables.

\section{Validation of the Nomogram}

The C-index of the nomogram was 0.724 (95\% CI, 0.683-0.765), which showed the nomogram provided good discernment. Additionally, the acceptable agreement between the predictions of nomogram and actual observations was illustrated by calibration plots for 3-, 5- and 10-year OS (Figure 3A-C). Moreover, time-dependent AUC with 6-fold cross-validation also showed consistent results (Figure 3D). These results indicated that the nomogram had proper calibration.

\section{Performance of the Nomogram in Risk Stratification of Patients}

The patients respective risk scores were calculated through the nomogram. The optimal cut-off total score was 194.9513. Then, all patients were assigned to the lowrisk group and the high-risk group on the basis of the cutoff score. As shown in Figure 4, overall survival of the low-risk group and the high-risk group is different $(P$-value $<0.0001)$.

\section{Discussion}

At present, neoadjuvant chemoradiotherapy is the standard treatment for LARC patients. However, individualized 
Table I Baseline Characteristics of Rectal Cancer Patients

\begin{tabular}{|c|c|}
\hline All Patients (n) & 911 \\
\hline \multicolumn{2}{|l|}{ Gender (n (\%)) } \\
\hline Female & $316(34.7)$ \\
\hline Male & $595(65.3)$ \\
\hline Age (years) (mean (SD)) & $55.30(11.33)$ \\
\hline \multicolumn{2}{|l|}{ Age (n (\%)) } \\
\hline$<60$ years & $571(62.7)$ \\
\hline$\geq 60$ years & $340(37.3)$ \\
\hline \multicolumn{2}{|l|}{ cT classification (n (\%)) } \\
\hline cT2 & $48(5.3)$ \\
\hline cT3 & $338(37.1)$ \\
\hline cT4 & $525(57.6)$ \\
\hline \multicolumn{2}{|l|}{ cN classification (n (\%)) } \\
\hline Negative & $90(9.9)$ \\
\hline Positive & $821(90.1)$ \\
\hline \multicolumn{2}{|l|}{ Pretreatment CEA (n (\%)) } \\
\hline Normal & $513(56.3)$ \\
\hline Elevated & $398(43.7)$ \\
\hline \multicolumn{2}{|l|}{ Pretreatment CAI99 (n (\%)) } \\
\hline Normal & $786(86.3)$ \\
\hline Elevated & $125(13.7)$ \\
\hline \multicolumn{2}{|l|}{ Location (n (\%)) } \\
\hline Low & $376(4 \mid .3)$ \\
\hline Middle & $464(50.9)$ \\
\hline High & 71 (7.8) \\
\hline \multicolumn{2}{|l|}{ Chemotherapy (n (\%)) } \\
\hline CapeOx & $232(25.5)$ \\
\hline Capecitabine & $524(57.5)$ \\
\hline 5-Fu based & $155(17.0)$ \\
\hline \multicolumn{2}{|l|}{ Radiotherapy (n (\%)) } \\
\hline 3DRT & $292(32.1)$ \\
\hline IMRT & $619(67.9)$ \\
\hline \multicolumn{2}{|l|}{ Surgery (n (\%)) } \\
\hline NO APR & $813(89.2)$ \\
\hline APR & $98(10.8)$ \\
\hline \multicolumn{2}{|l|}{ TLN (n (\%)) } \\
\hline$<12$ & $420(46.1)$ \\
\hline$\geq 12$ & $491(53.9)$ \\
\hline \multicolumn{2}{|l|}{ Posttreatment CEA (n (\%)) } \\
\hline Normal & $781(85.7)$ \\
\hline Elevated & $130(14.3)$ \\
\hline \multicolumn{2}{|l|}{ Posttreatment CAI99 (n (\%)) } \\
\hline Normal & $860(94.4)$ \\
\hline Elevated & $51(5.6)$ \\
\hline
\end{tabular}

(Continued)
Table I (Continued).

\begin{tabular}{|l|l|}
\hline All Patients (n) & 9 I I \\
\hline PT classification (n (\%)) & \\
PT0 & $187(20.5)$ \\
PTI & $55(6.0)$ \\
PT2 & $235(25.8)$ \\
PT3 & $377(41.4)$ \\
PT4 & $57(6.3)$ \\
\hline PN classification (n (\%)) & \\
PNO & $665(73.0)$ \\
PNI & $184(20.2)$ \\
PN2 & $62(6.8)$ \\
\hline PCR (n (\%)) & \\
NO & $733(80.5)$ \\
YES & $178(19.5)$ \\
\hline Adjuvant chemotherapy (n (\%)) & \\
NO & $198(21.7)$ \\
YES & $713(78.3)$ \\
\hline Status (n (\%)) & $736(80.8)$ \\
Censored & $175(19.2)$ \\
Death & $51.4(37.7,71.5)$ \\
Follow-up time (months, median(IQR)) & \\
\hline
\end{tabular}

Abbreviations: 3DRT, 3-dimensional conformal radiotherapy; IMRT, intensitymodulated radiation therapy; APR, abdominoperineal resection; TLN, the total number of examined lymph node; CEA, carcinoembryonic antigen; CAI9-9, carbohydrate antigen 19-9; IQR, interquartile range.

treatment may offer improved OS in high-risk rectal cancer patients. Thus, it is crucial to determine the proper prognostic factors. Currently, the TNM staging system is usually used to predict the individual survival of rectal cancer. ${ }^{11}$ However, there are still limitations in the accuracy of the TNM staging system. This study attempts to construct and validate a model to predict the prognosis of LARC patients who underwent nCRT.

Several studies identified CEA as a significant factor for predicting tumor response following nCRT and outcomes of rectal cancer. ${ }^{12-16}$ In this study, pretreatment CEA and posttreatment CEA were proved to be both of those prognostic factors. Pretreatment CEA also was a predictor for pCR (Figure S1). Multiple scholars reported that CA19-9 was good predictor of response to nCRT and survival after curative surgery of rectal cancer. ${ }^{10,17-19}$ This study also showed pretreatment CA19-9 and posttreatment CA199 were prognostic factors for overall survival of LARC patients with nCRT. 

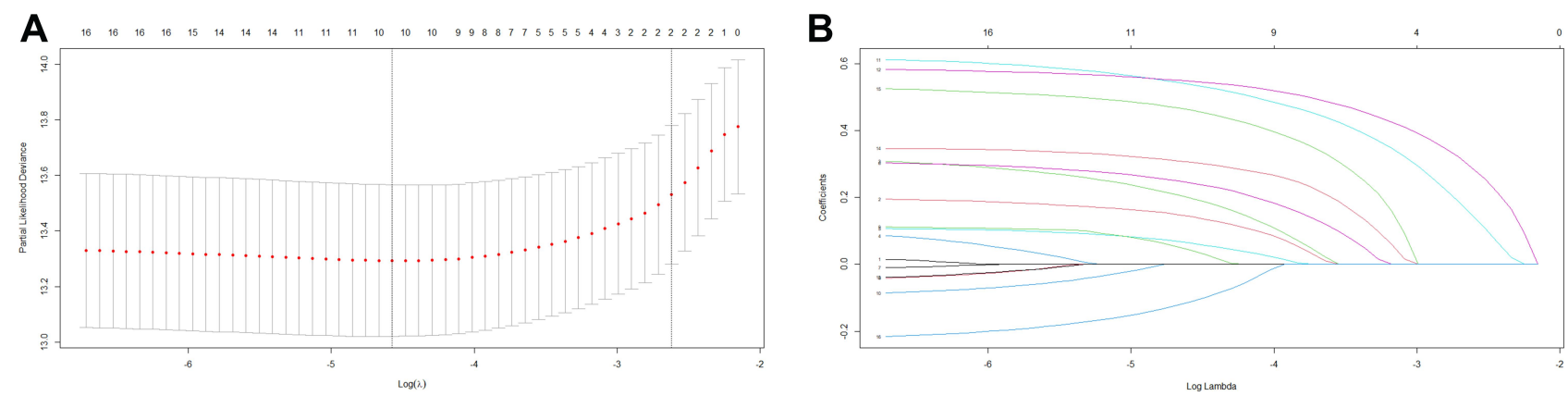

Figure I Feature selection. Selection of tuning parameter $(\lambda)$ in the LASSO regression (A), LASSO coefficient profiles for clinical features (B).

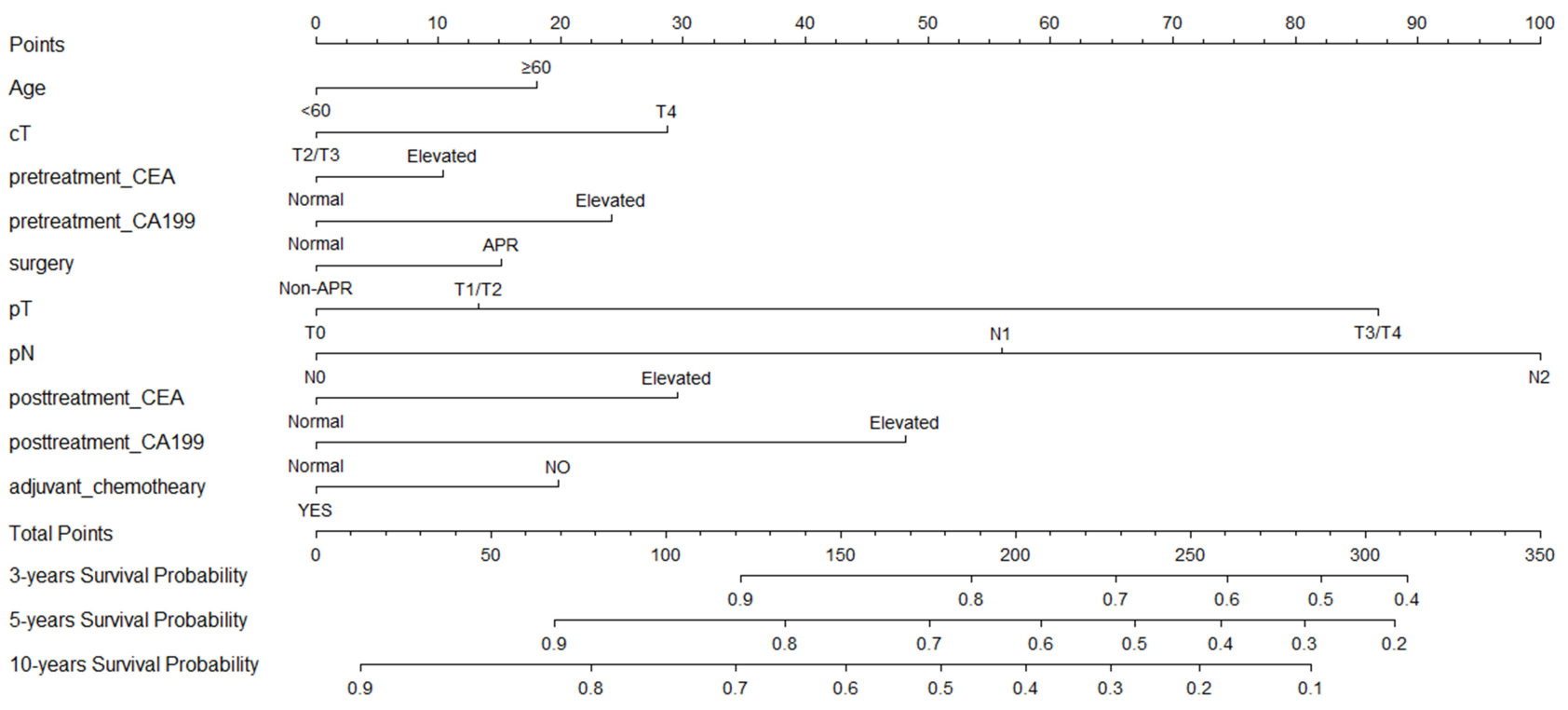

Figure 2 Nomogram for predicting 3-, 5-, and 10-year OS probabilities of rectal cancer patients. Draw a vertical straight line from the variable value to the axis labeled "Points". Then calculate all variables' points. The total points on the bottom scales that correspond to the 3-, 5-, and 10-year OS were showed apparently.

Some studies demonstrated that neoadjuvant chemoradiotherapy have decreased lymph node yield. ${ }^{20-22}$ Miller, E. D. et al showed that the decrease ranged from $7 \%$ to $53 \%$ in patients with nCRT. ${ }^{20}$ In Mechera, R. study, compared with patients without neoadjuvant treatment, patients who underwent nCRT yield 3.9 less lymph nodes. ${ }^{21} \mathrm{Ha}$, Y. H. et al showed that nCRT decreased retrieved lymph nodes count by approximately $33 \% .^{22}$ The minimum 12 lymph nodes examination was recommended by the College of American pathologists (CAP) and AJCC for accurate stages of rectal cancer. ${ }^{23-25}$ However, patients who received neoadjuvant treatment did not always achieve the examination of at least 12 lymph nodes. ${ }^{26-28} \mathrm{Xu}$, Z. et al showed that $36.8 \%$ of patients who underwent neoadjuvant treatment did not achieve 12 lymph nodes examined. ${ }^{26}$ Damin, D. C. et al showed that $29.6 \%$ of patients with nCRT achieved fewer than 12 lymph nodes. ${ }^{27}$ Baxter,
N. N. et al showed only $20 \%$ of patients treated with neoadjuvant therapy had at least 12 nodes sampling. ${ }^{28}$ In the study, $46.1 \%$ of patients did not achieve 12 lymph nodes examined. There were controversies about the impact of retrieved node counts on outcome of rectal cancer patients with nCRT. Ceelen, W.et al showed that per node examined got $2.7 \%$ decrease of hazard of death of in patients with surgery alone, no effect in patients with nCRT. ${ }^{29}$ Bhangu, A.et al showed that total lymph node yield was associated with overall survival in stage I and II rectal cancer, did not correlate with survival of stage ш patients. ${ }^{30}$ Sun, Y.et al showed that negative lymph nodes count was associated with disease-free survival in ypN + patients with nCRT. ${ }^{31}$ Hall, M. D. et al showed that examination of 8 or more LNs had better OS. ${ }^{32}$ Kim, H. J. et al showed that reduced lymph node yield after nCRT does not indicate inadequate oncologic surgery. ${ }^{33}$ Among patients with a good tumor 

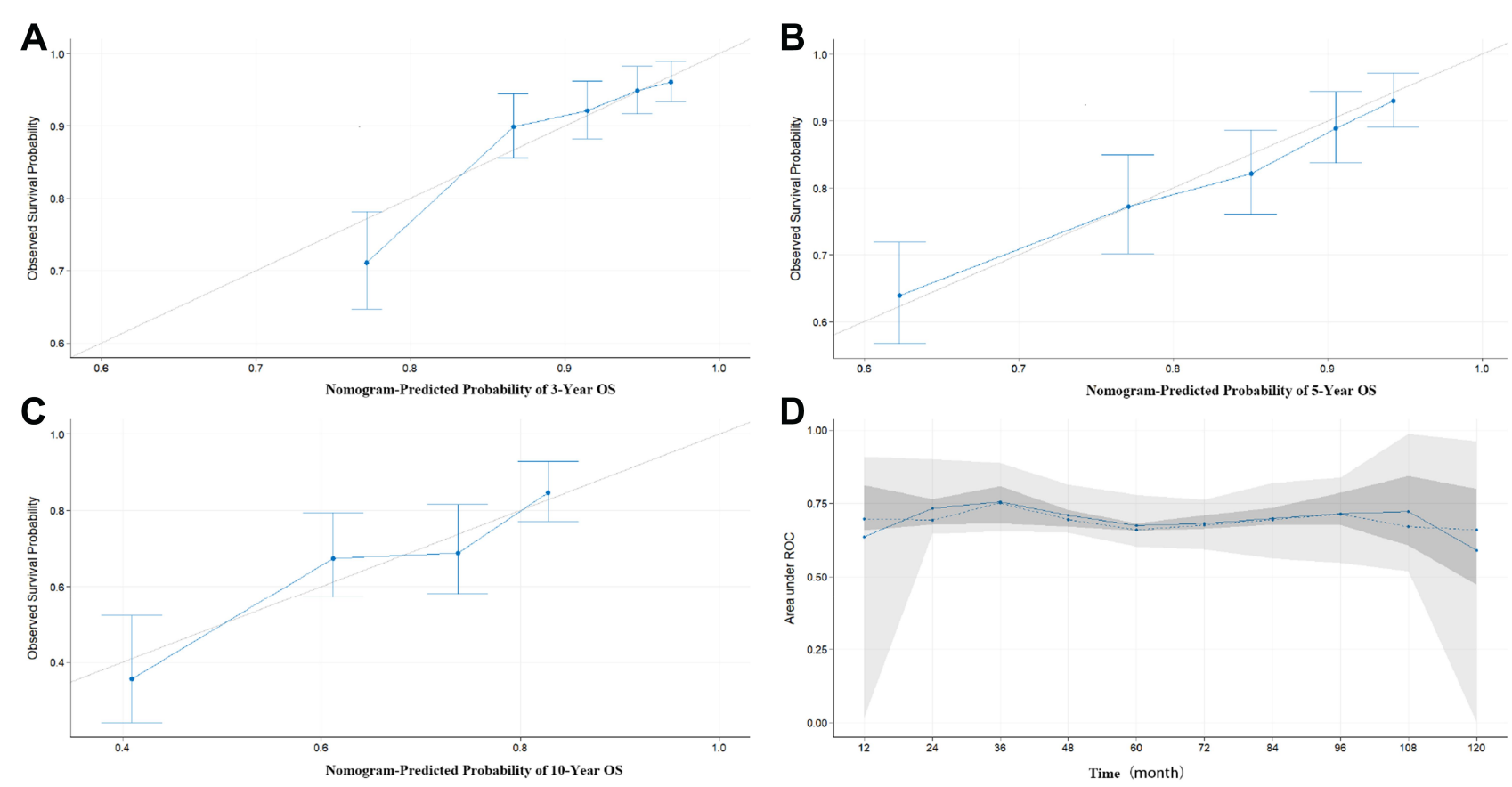

Figure 3 Calibration curves and time-dependent AUC. Calibration curves for predicting 3-, 5-, and I0-year OS (A, B, C). Time-dependent AUC with 6-fold crossvalidation (D).

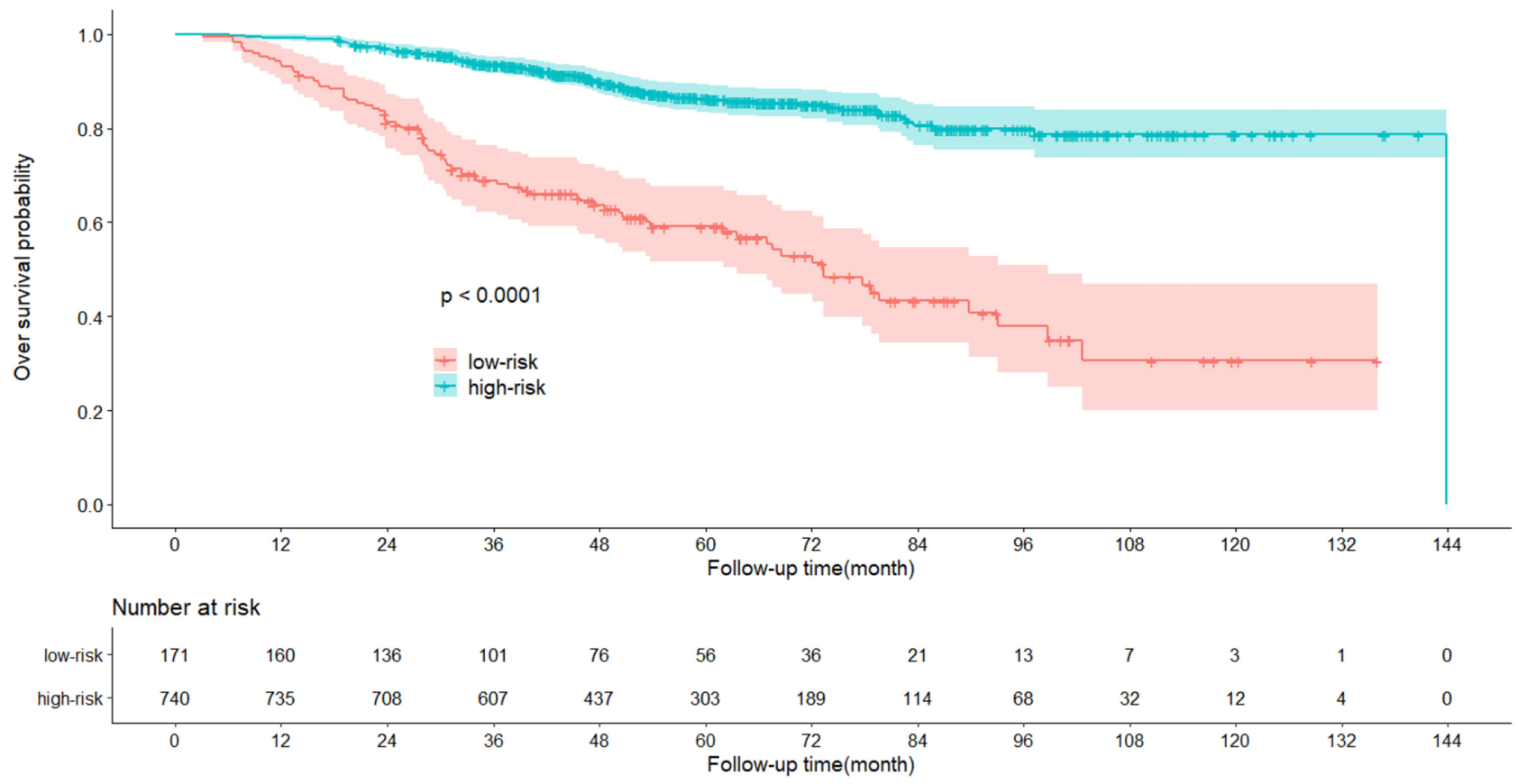

Figure 4 Kaplan-Meier curves of OS for patients in high-risk and low-risk groups.

response, patients with fewer than 12 lymph nodes got better 3-year disease-free survival. ${ }^{33}$ On the basis of CAP and AJCC, the cut-off of TLN still was 12 in the study. However, the present study showed TLN was not significant with the survival.
Nomograms with a graphical presentation of statistical prediction models are widely used as prognostic facilities in oncology and medicine. ${ }^{34}$ In the present study, ten characteristics were selected by LASSO regression analysis. The clinical prognostic indicators including CEA and 
CA199 were incorporated to set up the novel nomogram with better OS prediction for LARC patients. Clinical factors were applied for the nomogram, because of their widely and easily accessible. The nomogram was successfully set up to predict 3-, 5- and 10-year OS of LARC patients with nCRT. The nomogram illustrated that $\mathrm{pT}$ classification and $\mathrm{pN}$ classification contributes most to prognosis. The C-index was 0.724 , revealing the nomogram provided good discernment. Validation is a basic procedure in nomogram studies, which might avoid poor goodness-of-fit and assess the generalizability of the nomogram. ${ }^{35}$ In our study, the calibration curves of the 3-, 5- and 10-year survival fraction were executed. The calibration curves reveal favorable agreement between the predicted and actual observed probabilities. The calibration curve ensured the repeatability as well as the reliability of the constructed nomogram. The individual survival probability was conveniently calculated by clinicians and patients via this point-based nomogram. According to the total scores and the defined cut-off value, LARC patients with nCRT were stratified into highrisk and low-risk groups. The survival of these two groups was significantly different, which confirmed that the nomogram had a decent discriminatory capacity of the prognosis of patients and satisfactory predictive performance.

The nomogram was successfully constructed. And the nomogram was also validated to calculate the individual prognosis probability for LARC patients with nCRT. However, the study did have several limitations. Firstly, the study has selection bias because of retrospective nature of the analysis, in which some factors associated with survival were unavailable, such as smoking status, genetic alterations, lymphovascular invasion and so on. Secondly, due to the data are relatively small and from a single institution, these are some restriction on the applicable scope and generalizability of the nomogram. Thirdly, the nomogram is based on the experience of our single institution. It is necessary for more other institution external validations.

\section{Conclusion}

The nomogram is developed and validated to predict overall survival of resected LARC patients with nCRT. The proposed nomogram may help clinicians to perform individual treatment. Due to the limitations of the study, it is absolutely essential to further seek the unknown prognostic factors to optimize the model, and it is necessary for more other institution external validations.

\section{Funding}

The study is supported by the Natural Science Foundation of Fujian province (No. 2019J01149) and the Training project of Middle-aged and Young Core Talents in health system of Fujian Province (No.2019-ZQN-44).

\section{Disclosure}

The authors have no conflicts of interest to declare.

\section{References}

1. Torre LA, Bray F, Siegel RL, Ferlay J, Lortet-Tieulent J, Jemal A. Global cancer statistics, 2012. CA Cancer J Clin. 2015;65(2):87-108. doi: $10.3322 /$ caac. 21262

2. Kawai K, Sunami E, Yamaguchi H, et al. Nomograms for colorectal cancer: A systematic review. World $j$ Gastroenterol. 2015;21 (41):11877-11886. doi:10.3748/wjg.v21.i41.11877

3. Rodel C, Liersch T, Becker H, et al. Preoperative chemoradiotherapy and postoperative chemotherapy with fluorouracil and oxaliplatin versus fluorouracil alone in locally advanced rectal cancer: initial results of the German CAO/ARO/AIO-04 randomised Phase 3 trial. Lancet Oncol. 2012;13(7):679-687. doi:10.1016/S1470-2045(12) 70187-0

4. Sauer R, Becker H, Hohenberger W, et al. Preoperative versus postoperative chemoradiotherapy for rectal cancer. $N$ Engl J Med. 2004;351(17):1731-1740. doi:10.1056/NEJMoa040694

5. Sauer R, Liersch T, Merkel S, et al. Preoperative versus postoperative chemoradiotherapy for locally advanced rectal cancer: results of the German CAO/ARO/AIO-94 randomized Phase III trial after a median follow-up of 11 years. $J$ clin oncol. 2012;30(16):1926-1933. doi:10.1200/JCO.2011.40.1836

6. Liu Q, Lian P, Luo D, Cai S, Li Q, Li X. Combination of carcinoembryonic antigen with the American Joint Committee on Cancer TNM staging system in rectal cancer: a real-world and large population-based study. OncoTargets and Ther. 2018;2(11):5827-5834.

7. He Y, Ong Y, Li X, et al. Performance of prediction models on survival outcomes of colorectal cancer with surgical resection: A systematic review and meta-analysis. Surg Oncol. 2019;29:196-202. doi:10.1016/ j.suronc.2019.05.014

8. Glynne-Jones R, Wyrwicz L, Tiret E, et al. Rectal cancer: ESMO Clinical Practice Guidelines for diagnosis, treatment and follow-up. Ann Oncol. 2017;28(Suppl 4):iv22-iv40. doi:10.1093/annonc/mdx224

9. Xu B, Chen Y, Guo Y, et al. Pretreatment Tumor Thickness as a Predictor of Pathologic Complete Response to Neoadjuvant Chemoradiation Therapy for Stage II/III Rectal Adenocarcinoma. Am J Clin Oncol. 2018;41(6):601-606. doi:10.1097/COC.0000000000000333

10. Song J, Huang X, Chen Z, et al. Predictive value of carcinoembryonic antigen and carbohydrate antigen 19-9 related to downstaging to stage 0-I after neoadjuvant chemoradiotherapy in locally advanced rectal cancer. Cancer Manag Res. 2018;10:3101-3108. doi:10.2147/ CMAR.S166417

11. Siegel RL, Miller KD, Fedewa SA, et al. Colorectal cancer statistics, 2017. CA Cancer J Clin. 2017;67(3):177-193. doi:10.3322/caac.21395

12. Cai D, Huang ZH, Yu HC, et al. Prognostic value of preoperative carcinoembryonic antigen/tumor size in rectal cancer. World j Gastroenterol. 2019;25(33):4945-4958. doi:10.3748/wjg.v25.i33.4945

13. Wu S, Gu W. Association of T Stage and Serum CEA Levels in Determining Survival of Rectal Cancer. Fron Med. 2017;1(6):270. 
14. Kim CH, Huh JW, Yeom SS, Kim HR, Kim YJ. Predictive value of serum and tissue carcinoembryonic antigens for radiologic response and oncologic outcome of rectal cancer. Pathol Res Pract. 2020;216 (3):152834. doi:10.1016/j.prp.2020.152834

15. Nakamura Y, Shida D, Tanabe T, et al. Prognostic impact of preoperatively elevated and postoperatively normalized carcinoembryonic antigen levels following curative resection of stage I-III rectal cancer. Cancer Med. 2020;9(2):653-662. doi:10.1002/cam4.2758

16. Restivo A, Delrio P, Deidda S, et al. Predictors of Early Distant Relapse in Rectal Cancer Patients Submitted to Preoperative Chemoradiotherapy. Oncol Res Treat. 2020;7:1-7.

17. Yeo SG, Kim DY, Kim TH, et al. Carbohydrate antigen 19-9 levels associated with pathological responses to preoperative chemoradiotherapy in rectal cancer. Asian Pacif $j$ Cancer Prevent. 2014;15 (13):5383-5387. doi:10.7314/APJCP.2014.15.13.5383

18. Zhang LN, OuYang PY, Xiao WW, et al. Elevated CA19-9 as the Most Significant Prognostic Factor in Locally Advanced Rectal Cancer Following Neoadjuvant Chemoradiotherapy. Medicine. 2015;94(45):e1793. doi:10.1097/MD.0000000000001793

19. Miki H, Akiyoshi T, Ogura A, et al. Pretreatment Serum Carbohydrate Antigen 19-9 Concentration Is a Predictor of Survival of Patients Who Have Undergone Curative Resection of Stage IV Rectal Cancer. Dig Surg. 2018;35(5):389-396. doi:10.1159/ 000480247

20. Miller ED, Robb BW, Cummings OW, Johnstone PA. The effects of preoperative chemoradiotherapy on lymph node sampling in rectal cancer. Dis Colon Rectum. 2012;55(9):1002-1007. doi:10.1097/ DCR.0b013e3182536d70

21. Mechera R, Schuster T, Rosenberg R, Speich B. Lymph node yield after rectal resection in patients treated with neoadjuvant radiation for rectal cancer: A systematic review and meta-analysis. Eur j Cancer. 2017;72:84-94. doi:10.1016/j.ejca.2016.10.031

22. Ha YH, Jeong SY, Lim SB, et al. Influence of preoperative chemoradiotherapy on the number of lymph nodes retrieved in rectal cancer. Ann Surg. 2010;252(2):336-340. doi:10.1097/SLA.0b013e3181e61e33

23. Compton CC, Fielding LP, Burgart LJ, et al. Prognostic factors in colorectal cancer. College of American Pathologists Consensus Statement 1999. Arch Pathol Lab Med. 2000;124(7):979-994.

24. Sobin LH, Greene FL. TNM classification: clarification of number of regional lymph nodes for pNo. Cancer. 2001;92(2):452. doi:10.1002/ 1097-0142(20010715)92:2<452::AID-CNCR1342>3.0.CO;2-B
25. NCCN Clinical Practice Guidelines in Oncology Rectal Cancer. Version 1, 2020.Available at:. https:/wwwnccnorg/professionals/phy sician_gls/pdf/rectalpdf.

26. $\mathrm{Xu} \mathrm{Z}$, Berho ME, Becerra AZ, et al. Lymph node yield is an independent predictor of survival in rectal cancer regardless of receipt of neoadjuvant therapy. J Clin Pathol. 2017;70(7):584-592. doi:10.1136/jclinpath-2016-203995

27. Damin DC, Rosito MA, Contu PC, et al. Lymph node retrieval after preoperative chemoradiotherapy for rectal cancer. $J$ Gastrointest Surg. 2012;16(8):1573-1580. doi:10.1007/s11605-012-1916-4

28. Baxter NN, Morris AM, Rothenberger DA, Tepper JE. Impact of preoperative radiation for rectal cancer on subsequent lymph node evaluation: a population-based analysis. Int J Radiat Oncol Biol Phys. 2005;61(2):426-431. doi:10.1016/j.ijrobp.2004.06.259

29. Ceelen W, Willaert W, Varewyck M, Libbrecht S, Goetghebeur E, Pattyn P. Effect of neoadjuvant radiation dose and schedule on nodal count and its prognostic impact in stage II-ш Rectal Cancer. Ann Surg Oncol. 2016;23(12):3899-3906. doi:10.1245/s10434-016-5363-4

30. Bhangu A, Kiran RP, Brown G, Goldin R, Tekkis P. Establishing the optimum lymph node yield for diagnosis of stage III rectal cancer. Tech Coloproctol. 2014;18(8):709-717. doi:10.1007/s10151-013-1114-8

31. Sun Y, Zhang Y, Huang Z, Chi P. Prognostic implication of negative lymph node count in ypN+ rectal cancer after neoadjuvant chemoradiotherapy and construction of a prediction nomogram. J Gastrointest Surg. 2019;23(5):1006-1014. doi:10.1007/s11605-018-3942-3

32. Hall MD, Schultheiss TE, Smith DD, et al. Impact of total lymph node count on staging and survival after neoadjuvant chemoradiation therapy for rectal cancer. Ann Surg Oncol. 2015;22(Suppl 3):S580S587. doi:10.1245/s10434-015-4585-1

33. Kim HJ, Jo JS, Lee SY, Kim CH, Kim YJ, Kim HR. Low lymph node retrieval after preoperative chemoradiation for rectal cancer is associated with improved prognosis in patients with a good tumor response. Ann Surg Oncol. 2015;22(6):2075-2081. doi:10.1245/ s10434-014-4235-z

34. Balachandran VP, Gonen M, Smith JJ, DeMatteo RP. Nomograms in oncology: more than meets the eye. Lancet Oncol. 2015;16(4):e173e180. doi:10.1016/S1470-2045(14)71116-7

35. Iasonos A, Schrag D, Raj GV, Panageas KS. How to build and interpret a nomogram for cancer prognosis. J clin oncol. 2008;26 (8):1364-1370. doi:10.1200/JCO.2007.12.9791

\section{Publish your work in this journal}

Cancer Management and Research is an international, peer-reviewed open access journal focusing on cancer research and the optimal use of preventative and integrated treatment interventions to achieve improved outcomes, enhanced survival and quality of life for the cancer patient.
The manuscript management system is completely online and includes a very quick and fair peer-review system, which is all easy to use. Visit http://www.dovepress.com/testimonials.php to read real quotes from published authors. 